\title{
ADVANCED METHODS OF HEART RATE SIGNALS PROCESSING AND THEIR USEFULNESS IN DIAGNOSIS SUPPORT II. UNIVARIATE STATISTICAL TECHNIQUES
}

\author{
JERZY A. MOCZKO \\ Chair and Department of Computer Science and Statistics \\ University of Medical Sciences in Poznań \\ Dabrowskiego 79, 60-529 Poznań, Poland \\ e-mail address: jmoczko@am.poznan.pl
}

(Rec. 29 February 2004)

\begin{abstract}
A tentative cardiological database was established using virtual instrumentation described in the first part of presented paper. Some additional not heart rate variability parameters were added. Three selected univariate statistical techniques were used for illustration diagnosis support techniques in discrimination between healthy and coronary heart disease people. Comparison of nonparametric MannWhitney test, receiver operating characteristic ROC analysis and univariate logistic regression results was performed. In all used methods long term heart rate variability indices were most useful in prediction of patient's status. The correctness of classification was between 55 to 79 percent with ROC technique and 68 to 78 percent with logistic regression. However high number of false negative FN cases excludes univariate techniques as reliable screening test.
\end{abstract}

\section{INTRODUCTION}

In first part of the paper a wide set of heart rate variability (HRV) indices in time, frequency and time-frequency domains was described [1]. The specially designed virtual instrumentation allows for interactive choice of ECG segment of interest (SOI) in anyone of 12-leads, detects $R$-waves, estimates $T_{R-R}$ intervals and heart rate (HR), calculates HRV indices and finally saves them in ASCII format database. This database was supplemented with additional information about patients. Whereas all data obtained from signal analysis are measured in statistical interval scale, these last are expressed in interval, ordinal or nominal scale. The first scale is represented by age, body mass index BMI, systolic RR1 and diastolic RR2 blood pressure, duration of the heart disease, echocardiographic measurements (left ventricle LK, left atrium LP, right ventricle PK, ventricular septum PMK, posterior wall left ventricle TSLK), lipids levels (total cholesterol, high- and low-density cholesterol, triglycerides levels), electrolytes levels (sodium and potassium), glucose and glycolysed hemoglobin HBA1C. In ordinal scale two parameters are measured contractility and coronary angiography results. Gender, pharmacological treatment, family history, other diseases and tobacco smoking are the measurements expressed in nominal scale. In presented preliminary methodological studies 61 data records were analyzed: 19 patients with diabetes, 27 with 
coronary heart disease (CHD) and 15 healthy patients. The last group consisted of volunteers both age- and sex-matched. Clinical symptoms of CHD were confirmed with coronary angiography. ECG was recorded in five following situations: in supine position, in standing position, in supine position after administration of single-dose one milligram of atropine subcutaneously, in supine position after administration of single oral dose 40 milligrams of propranolol and in supine position after administration of single oral dose 25 milligrams of captopril. The main goal of presented part of study is to compare properties of measured parameters in discrimination between patients with stable coronary heart disease and control group in univariate approach.

\section{DESCRIPTIVE AND CLASSICAL HYPOTHESIS TESTING STATISTICAL RESULTS}

In the first step of statistical analysis normal distribution of interval scale parameters in both analyzed data groups was checked. Lillierforce's and Shapiro-Wilk's tests revealed not Gaussian distribution of data in prevalent number of measured parameters. On the other hand Levene test demonstrated lack of homogeneity of variances. These results and relatively small sample sizes forced us to use nonparametric Mann-Whitney test as a nonparametric alternative to the t-test for independent samples [2]. High power of Mann-Whitney test $(95.5 \%$ in comparison to t-Student test) does not change substantially the ability of distinction between central tendency measures in analyzed groups. All mentioned calculations were done with StatSoft STATISTICA Data Miner version 6.1 (2004). The example of obtained results for standing position is presented in Fig. 1.

\begin{tabular}{|c|c|c|c|c|c|c|c|c|c|c|}
\hline \multirow[b]{2}{*}{ fansth } & \multicolumn{10}{|c|}{ 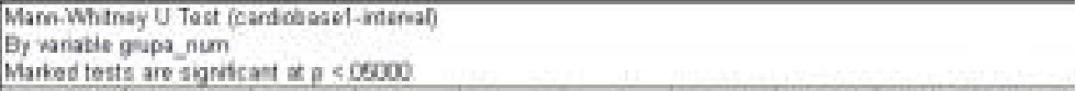 } \\
\hline & $\begin{array}{l}\text { Hanksum } \\
\text { Croup } 1\end{array}$ & $\begin{array}{l}\text { Amb sum } \\
\text { Goup? }\end{array}$ & u & Z & phent & $\begin{array}{c}\text { I } \\
\text { nturded }\end{array}$ & Peres & $\begin{array}{l}\text { Yald } 14 \\
\text { Groue } 1\end{array}$ & $\begin{array}{l}\text { Yadid } 14 \\
\text { Gmup } 2\end{array}$ & $\begin{array}{l}\text { Fluded } \\
\text { Findp }\end{array}$ \\
\hline PI rili II & 39000 & 4540000 & 113000 & 221515 & OM.fit5 & 22155 & ․ㅗ451 & 15 & 25 & DOSE14: \\
\hline PL_Olian FAM & 419 ming & 448000 & 9700 & 2 fivint & on mpre & $26 x$ & D IITTMal & 15 & 76 & $100 \pi 1^{1}$ \\
\hline PLer Gen 0 & si 000 & 400.0010 & 12000 & 1 robu & oniate & 170840 & Dopati & 15 & IS. & ogot 1 \\
\hline Pl_Huly $9 T$ \% & 3900000 & 4E8.0000 & 1170000 & 211119 & DISATE & 211119 & DIJUISE & 15 & $\pi$ & 1034813 \\
\hline PIHIEY LT & 4290ี0 & 450070 & 61 1000 & 30057 & 00 Ton & 30659 & Domes: & 15 & $x$ & $1005=$ \\
\hline PID Dvtae WAES & 38000 & 520000 & 17000 & IEASBI & 051591 & D6490. & 0.54542 & Is & x & 15.3161 \\
\hline PIDAlus ED & 3720000 & 4990070 & t3acono & 1548 & $0+x \in a$ & 1547801 & C. 172851 & 15 & $x$ & a17726? \\
\hline PI_zugab STY & saboo & 40000 & 142000 & 1445 & 101422 & 1.4584 & 0.161951 & $\$ 5$ & $x$ & 0.590 \\
\hline
\end{tabular}

Fig. 1. Fragment of Mann-Whitney test results for standing position - comparison between coronary heart disease and control groups

The prominent distinction between CHD and healthy people was observed in all five clinical situations in Huey LTV index and in w(2) wavelet-transform standard deviation. Four indices (Organ BAND, oscillation index OSC and w(1) and w(3) wavelet-transform standard deviations detected the difference after captopril and propranolol administration and in 
Table 1. Usefulness of analyzed indices in discrimination between CHD and healthy people using nonparametric Mann-Whitney test in five different clinical situations. Plus sign denotes statistical significance at least at 0.05

\begin{tabular}{|c|c|c|c|c|c|}
\hline Index & Supine Position & Standing Position & Captopril & Atropine & Propranolol \\
\hline \multicolumn{6}{|l|}{ De Haan STI } \\
\hline De Haan LTI & & & & & + \\
\hline \multicolumn{6}{|l|}{ Yeh DI } \\
\hline Yeh II & & + & & & + \\
\hline Organ BAND & & + & + & & + \\
\hline van Geijn ID & & & & + & + \\
\hline Huey STV & & + & & & + \\
\hline Huey LTV & + & + & + & + & + \\
\hline \multicolumn{6}{|l|}{ Dalton MABB } \\
\hline Dalton DSD & & & & & + \\
\hline \multicolumn{6}{|l|}{ Zugaib STV } \\
\hline Zugaib LTV & & + & & & + \\
\hline $\mathrm{OSC}$ & & + & + & & + \\
\hline SDNN & & & & & + \\
\hline RMSSD & + & & + & & \\
\hline Allan & & + & & & 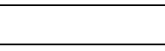 \\
\hline FFT total power & & + & & & + \\
\hline \multicolumn{6}{|l|}{ FFT VLF } \\
\hline \multicolumn{6}{|l|}{ FFT LF } \\
\hline \multicolumn{6}{|l|}{ FFT HF } \\
\hline \multicolumn{6}{|l|}{ FFT LF/HF } \\
\hline $\mathrm{w}(1)$ & & + & & + & + \\
\hline $\mathrm{w}(2)$ & + & + & + & + & + \\
\hline$w(3)$ & + & & + & & + \\
\hline \multicolumn{6}{|l|}{$w(4)$} \\
\hline$w(5)$ & & + & & & \\
\hline $\mathrm{AR}(1)$ & & & & & + \\
\hline $\operatorname{AR}(2)$ & & + & & & \\
\hline \multicolumn{6}{|l|}{$\operatorname{AR}(3)$} \\
\hline \multicolumn{6}{|l|}{$\operatorname{AR}(4)$} \\
\hline \multicolumn{6}{|l|}{$\operatorname{AR}(5)$} \\
\hline \multicolumn{6}{|l|}{$\operatorname{AR}(6)$} \\
\hline \multicolumn{6}{|l|}{$\operatorname{AR}(7)$} \\
\hline \multicolumn{6}{|l|}{$\mathrm{AR}(8)$} \\
\hline \multicolumn{6}{|l|}{$\operatorname{AR}(9)$} \\
\hline \multicolumn{6}{|l|}{$\mathrm{AR}(10)$} \\
\hline \multicolumn{6}{|l|}{$\operatorname{AR}(11)$} \\
\hline \multicolumn{6}{|l|}{$\operatorname{AR}(12)$} \\
\hline \multicolumn{6}{|l|}{ AR exc.noise } \\
\hline AR total power & & & & & + \\
\hline \multicolumn{6}{|l|}{ AR ULF } \\
\hline \multicolumn{6}{|l|}{ AR VLF } \\
\hline AR LF & & & + & & + \\
\hline AR HF & & & & & + \\
\hline AR LF/HF & & & + & & + \\
\hline
\end{tabular}


standing position. We did not found statistically significant difference in all short term variability indices (de Haan STI, Yeh DI, Dalton MABB, Zugaib STV and in majority of nonparametric frequency domain indices. In Table 1 the details of univariate approach are presented. On similar principle different clinical experiments may be performed. For example one may treat particular index value in supine position before drug administration as an initial status and compare the statistical significance of differences between this status and status after $\beta$-blockade in different heart diseases.

\section{RECEIVER OPERATING CHARACTERISTIC CURVES}

In the very majority of clinical situations we want to require a yes/no decision on the presence of a disease. This decision is done by comparing the output value of clinical test to some particular threshold. If obtained result of the test is above the threshold, the test is said to be positive (i.e. the disease is present, true positive TP). If the output is below the threshold, the test is said to be negative (i.e. the disease is absent, true negative TN). Of course, the opposite direction of decision making may be established, too. If the threshold unambiguously divides region of these two possible situations (healthy versus ill), we have no problem in diagnosis support. However, in real life high variability of variables describing actual status of living beings makes the discrimination problem more complex. The corresponding probability distribution functions ( $\mathrm{pdf}$ ) of measured parameters frequently overlap and unambiguous threshold does not exist. Figure 2 presents exemplary probability distribution functions for Zugaib's LTV index after propranolol administration for healthy and CHD people.

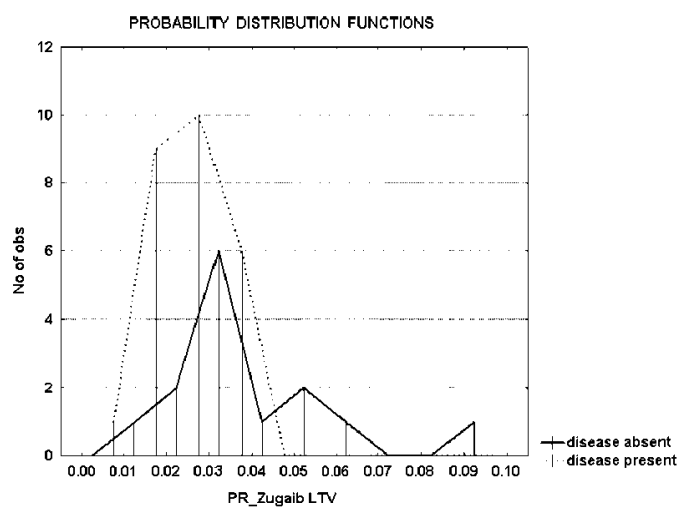

Fig. 2. Probability distribution functions for Zugaib's long term variability index after propranolol administration in control and coronary heart disease group

It is easy to find, that there does not exist any threshold level which uniquely discriminates mentioned groups of patients. We observe false positive results FP (when diagnostic test result is positive i.e. abnormal and true subject's condition is negative i.e. normal) or false negative results FN (in inverse situation). Statisticians introduce some indices to quantify quality of lab test: sensitivity (fraction of all cases with the disease who get a positive test result) and 
specificity (fraction of all cases without the disease who get a negative test result). In other words sensitivity measures the ability of the test to detect the disease, while specificity describes the ability to exclude those objects who do not have the disease. Both indices belong to the interval $\langle 0,1\rangle$. Optimal situation is when sensitivity and specificity are equal to one. It corresponds to the situation when number of FP and FN cases is equal to zero (pdf curves do not overlap). The plot of sensitivity as a function of (1-specificity) is known as Receiver Operating Characteristic (ROC) curve [3, 4]. If the curve does not significantly differ from diagonal line (area under ROC curve does not significantly differ from 0.5 ) examined lab test does not effectively discriminate compared groups. Full discrimination is obtained when area under the curve is equal to one (number of FP and FN is equal to zero). Figure 3 presents results of ROC analysis obtained for Zugaib's long term variability index after propranolol administration in control and coronary heart disease group estimated with Analyse-It Software, ver. 1.62. $p$-value, which is less than assumed significance level $\alpha=0.05$ confirms, that Zugaib's LTV index may be successfully used for CHD detection after propranolol administration. This result is consistent to Mann-Whitney test results in Table 1.

\begin{tabular}{|c|c|c|c|c|c|}
\hline Curve & Area & SE & p & 95F CI of Ared & grupa num $=2$ \\
\hline PR_Zugaib LTV & 0.821 & 0.0703 & 40.0001 & 0.684 to 0.959 & houe lomet values \\
\hline
\end{tabular}
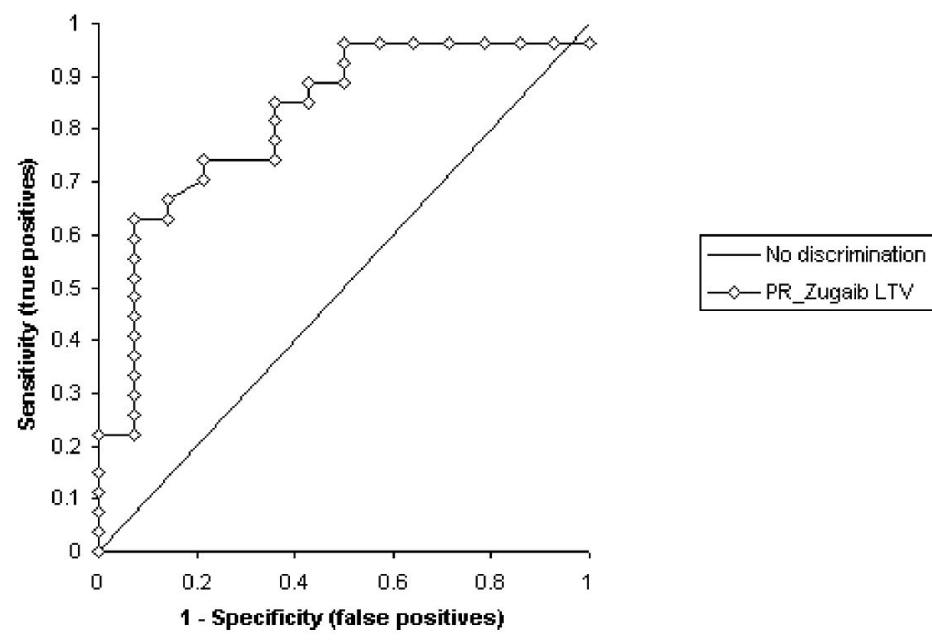

Fig. 3. ROC curve for Zugaib's long term variability index after propranolol administration in control and coronary heart disease group

In hypothesis testing theory the decrement of first type error increases the second type error (and vice versa) when sample size is constant. We have analogous situation in diagnosis 
support. Shifting the threshold level in the range of examined parameter we change number of FP and FN cases. We may follow two different ways:

1. find a threshold level which simultaneously maximizes both sensitivity and specificity indices or

2. find a threshold level which corresponds to parameter value assuring assumed misclassification ratio.

\begin{tabular}{|c|c|c|c|c|c|c|}
\hline 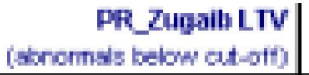 & Snsithoy & Sperificity & TP & $\mathrm{TH}$ & $\mathrm{FP}$ & $\mathbf{F H}$ \\
\hline Donas & $0.0 \%$ & 10008 & 9 & 14 & [0 & 27 \\
\hline 0.0127 & 3.74 & 10008 & 1 & 14 & 0 & 26 \\
\hline 0.0130 & $7,4 \%$ & $100.0 \%$ & 2 & 14 & 0 & 25 \\
\hline 0.0135 & $11.1 \mathrm{~K}$ & $100.0 \%$ & 3 & 14 & 0 & 24 \\
\hline 0.0153 & $14.8 \%$ & 100.08 & 4 & 14 & 0 & 23 \\
\hline 0.0157 & 2224 & 10008 & 6 & 14 & 0 & 21 \\
\hline 0.0163 & $22.2 \%$ & 9296 & 6 & 13 & 1 & 21 \\
\hline 0.0166 & $25.9 \%$ & 92.96 & $T$ & 13 & 1 & 20 \\
\hline 0.0170 & $28.6 \%$ & 9295 & 6 & 13 & 1 & 18 \\
\hline 00198 & 33.34 & $929 \%$ & 9 & 13 & 1 & 18 \\
\hline 0.0209 & $37.0 \%$ & $929 \%$ & 10 & 13 & 1 & 17 \\
\hline 0.0216 & $40.7 \%$ & 9296 & 11 & 13 & 1 & 16 \\
\hline 00221 & $44.4 \%$ & 9295 & 12 & 13 & 1 & 15 \\
\hline 0.0225 & $48,1 \%$ & 9294 & 13 & 13 & 1 & 14 \\
\hline 0.0227 & $51.9 \%$ & 92.96 & 14 & 13 & 1 & 13 \\
\hline 0.0238 & $55.6 \%$ & 9295 & 15 & 13 & 1 & 12 \\
\hline 00262 & 59.34 & 9294 & 16 & 13 & 1 & 11 \\
\hline 0.0264 & $630 \%$ & 9298 & 17 & 13 & 1 & 10 \\
\hline 0.0205 & $63.0 k$ & $95.7 \%$ & $1 \mathrm{~T}$ & 12 & 2 & 10 \\
\hline 0.0263 & $66.7 x$ & 65.75 & 16 & 12 & 2 & 9 \\
\hline 10त20日 & 7048 & 78,64 & 19 & 11 & 3 & B \\
\hline 0.0911 & $74.1 \%$ & TBGS & 20 & 11 & 3 & 7 \\
\hline 0.0314 & $74.1 \%$ & 64.98 & 20 & 9 & $\overline{5}$ & $\overline{7}$ \\
\hline 0.0323 & 77.84 & 6435 & 21 & g. & 5 & 6 \\
\hline 0.0939 & 81.54 & 6434 & 22 & g. & 5 & 5 \\
\hline 0.0362 & 85.26 & 8436 & 23 & g) & 5 & 4 \\
\hline 0.0366 & $05.2 \mathrm{~K}$ & $57.1 \%$ & 23 & - & 6 & 4 \\
\hline
\end{tabular}

Fig. 4. Sensitivity and specificity values as a function of threshold level for Zugaib's long term variability index after propranolol administration in control and coronary heart disease group (fragment of results)

The second situation is used when first and second type errors have not equivalent weight (i.e. it is better to detect the disease in healthy patient in a screening test than treat sick person as healthy one). In Fig. 4. we present sensitivity and specificity values obtained for different threshold level. Highlighted threshold 0.0311 maximizes simultaneously sensitivity and specificity and it may be used as "gold standard" if we assume that both types of mis- 
classification are equivalently dangerous. We must remember that "gold standard" estimation should be confirmed by results obtained from sufficient sample size.

\begin{tabular}{|c|c|c|c|c|c|c|c|c|c|}
\hline 1 & Parameter & Cutaff & Sensithity & Specificity & TP & $\mathrm{TN}$ & FP & $\mathrm{FN}$ & STATUS \\
\hline 2 & Ogan BAND & 6.0225 & $77.6 \%$ & $600 \%$ & 21 & 9 & 5 & 5 & SUPINE \\
\hline 3 & Husy LTV & 047644 & $667 \%$ & BDob & 18 & 12 & 3 & 9 & SUPINE \\
\hline 4 & Zugaib LTV & 0.0299 & $067 \%$ & 8006 & 18 & 9 & 8 & 9 & SUPINE \\
\hline 5 & OSC & 128852 & $70.4 \%$ & $B E . T S$ & 19 & 10 & 5 & 8 & SLPINE \\
\hline 6 & RMSSD & 47.7956 & $60.0 \%$ & $1000 \%$ & 17 & 15 & 0 & 10 & SUPINE \\
\hline 7 & ALLAN & 0.1213 & $66.7 \%$ & 7335 & 18 & 11 & 4 & 9 & SUIPINE \\
\hline 8 & FFT TOTAL PON & 7.3602 & $70.4 \%$ & $600 \%$ & 18 & $g$ & 6 & 8 & SUPINE \\
\hline 9 & $W_{2}$ & 27260 & $741 \%$ & 6E. T\% & 20 & 10 & 5 & 7 & SUPINE \\
\hline 10 & Wa & 3.0031 & $74.1 \%$ & $600 \%$ & 20 & 9 & 6 & 7 & SULPINE \\
\hline 11 & YEH II & 0,0459 & $04.6 \%$ & 66.75 & 22 & 10 & 5 & 4 & STANDNG \\
\hline 12 & Organ BAND & 7.1106 & $92.3 \%$ & $600 \%$ & 24 & 9 & 6 & 2 & STANDING \\
\hline 13 & van Gojin ID & 4.9467 & $73.1 \%$ & $600 \%$ & 19 & 9 & 6 & 7 & STMNDING \\
\hline 14 & Huey STV & 104.5 .12 & $78.9 \%$ & BE.TS & 20 & 10 & 5 & $\bar{B}$ & STANDING \\
\hline 15 & Huey LTV & 109.1154 & $76.9 \%$ & 0006 & 20 & 12 & 3 & 6 & STANDING \\
\hline 16 & Zugaib LTV & 0.0557 & $00.0 \%$ & 66.75 & 21 & 10 & 5 & 5 & STANDING \\
\hline 17 & OSc & 15.5660 & $04.6 \%$ & 6675 & 22 & 10 & 5 & 4 & STANDNG \\
\hline 10 & SDNW & 17.7806 & $73.1 \%$ & $6 \overline{6} 75$ & 19 & 10 & 5 & 7 & STANDIVG \\
\hline 19 & $A \perp A N$ & 0.1094 & $57.7 \%$ & $1000 \%$ & 15 & 15 & 0 & 11 & STANDING \\
\hline 20 & FFT TOTAL POWH & 8.3805 & $73.1 \%$ & BETS & 19 & 10 & 5 & 7 & STANDING \\
\hline 21 & W1 & 1.0437 & $69.2 \%$ & 06.TS & 18 & 13 & 2 & 8 & STANDNG \\
\hline 22 & $M 2$ & 3.1160 & $00.5 \%$ & 66.75 & 23 & 10 & 5 & 3 & STANDNG \\
\hline 23 & 105 & 4.5053 & $80.8 \%$ & 6005 & 21 & 8 & 6 & 5 & STANDNG \\
\hline 24 & AR EXC. Noise & 0,1327 & $731 \%$ & 5335 & 10 & 8 & 7 & 7 & STANDIV \\
\hline 25 & Drgan BAND & 3.5488 & $030 \%$ & 7336 & 17 & 11 & 4 & 10 & CAPTOPRIIL \\
\hline 26 & van Gejin ID & 20780 & $51.9 \%$ & 6006 & 14 & 9 & 6 & 13 & CAPTOPPRIL \\
\hline 27 & Huey LTV & 54.9069 & $66.7 \%$ & BE.TS & 18 & 10 & 5 & 9 & CAPTOPRIL \\
\hline 28 & $\mathrm{OSC}$ & 0.4879 & $66.7 \%$ & 8005 & 18 & 12 & 3 & 9 & CAPTOPRIL \\
\hline 29 & RMSSD & 397928 & $01.5 \%$ & 6005 & 22 & 9 & 6 & 5 & CAPTOPRIL \\
\hline 30 & FFT TOTAL PON & 3.7616 & $74.1 \%$ & 6Б) T\$ & 20 & 10 & 5 & 7 & CAPTOPRIL \\
\hline 31 & LF & 0.4292 & $630 \%$ & $600 \%$ & 17 & 9 & 6 & 10 & CAPTOPRIII \\
\hline 32 & 12 & 1.6347 & $77.8 \%$ & 7336 & 21 & 11 & 4 & $E$ & CAPTOPRIL \\
\hline 33 & Wa & 2.4349 & $65.4 \%$ & 73.36 & 17 & 11 & 4 & 9 & CAPTOPRIL \\
\hline 34 & Wi & 2.0850 & $57.7 \%$ & 0005 & 15 & 12 & 3 & 11 & CAPTOPRIL \\
\hline
\end{tabular}

Fig. 5. Threshold levels, corresponding sensitivity, specificity, number of true positives TP, true negatives TN, false positives FP and false negative FN cases in different clinical status-discrimination between CHD and control groups

In Fig. 5 and Fig. 6. we present the gathered results for ROC analysis of examined patients. One can find that the proper classification belongs to the interval $\langle 55,79\rangle$ percent, with mean value $76 \%$ after propranolol administration, 73\% - in standing position, $70 \%$ - in supine position, $69 \%$ - after atropine administration and $63 \%$ - after captopril administration. In each clinical situation we observe prevalent number of FN cases in misclassification, what should 


\begin{tabular}{|c|c|c|c|c|c|c|c|c|c|}
\hline 1 & Parameter & Cutoff & Sensithty & Sperificity & TP & $\mathbf{T N}$ & $\mathrm{FP}$ & $\mathrm{FH}$ & STATUS \\
\hline 35 & AR TOTAL POW & 16.0632 & $66.7 \%$ & $53.2 \%$ & 10 & B & 7 & 9 & CAPTOPRIL \\
\hline 36 & $A R L F$ & 0.2757 & $59.3 x$ & $66.7 \%$ & 16 & 13 & 2 & 11 & CAPTOPRIL \\
\hline 37 & AR HF & 0.10202 & $50.3 x$ & $60.0 \%$ & 16 & 9 & 6 & 11 & CAPTOPRIL \\
\hline 38 & AR LFMF & 0.4997 & $59.3 *$ & $800 \%$ & $1 \mathrm{~B}$ & 12 & 3 & 11 & CAPTOPRIL \\
\hline 39 & Organ BAND & 6..3784 & $778 x$ & $60.0 \%$ & 21 & 9 & 6 & 6 & ATPOAPNE \\
\hline 40 & wan Geijn ID & 3.9016 & 63.0\% & $60.7 \%$ & 17 & 10 & 5 & 10 & ATROPNE \\
\hline 41 & Huey STV & 1006193 & $63.0 \%$ & $60.0 \%$ & 17 & 9 & 6 & 10 & ATROPNE \\
\hline 42 & Huey LTV & 121.5879 & $61.5 \%$ & $66.7 \%$ & 22 & 10 & 5 & 5 & ATROPNE \\
\hline 43 & $08 \mathrm{C}$ & 14.6160 & $70.4 \%$ & $66.7 \%$ & 19 & 10 & 5 & 8 & ATPOFNE \\
\hline 44 & FNSSD & 45.9777 & $630 \%$ & $60.7 \%$ & 17 & 10 & 5 & 10 & ATROPIE \\
\hline 45 & FFT TOTAL PON & 7.1042 & $70.4 \%$ & $60.0 \%$ & 19 & 9 & 6 & 8 & ATROPIE \\
\hline 46 & W1 & 1.3987 & $81.5 \%$ & $60.7 \%$ & 22 & 10 & 5 & 5 & ATROPNE \\
\hline 47 & Wh & 2.7912 & $70.4 \%$ & $66.7 \%$ & 19 & 10 & 5 & 日 & ATROPNE \\
\hline 48 & de Haan LTI & 57,0547 & $77.8 \%$ & $85.7 \%$ & 21 & 12 & 2 & 6 & PROPRAN \\
\hline 49 & VEH DI & 0,0150 & $58.3 \%$ & $71.4 \%$ & 16 & 10 & 4 & 11 & PROPRAN \\
\hline 50 & Y'EH II & 0.0990 & $74,1 \%$ & $85.7 \%$ & 20 & 12 & 2 & 7 & PROPRAN \\
\hline 51 & Organ BAND & 4.4529 & $70.4 \%$ & $92.9 \%$ & 19 & 13 & 1 & 8 & PROPFAAN \\
\hline 52 & wan Geijn ID & 4.6170 & $91.5 \%$ & $71.4 \%$ & 22 & 10 & 4 & 5 & PROPRAN \\
\hline 53 & Huey STY & 1012665 & $81.5 \%$ & $71.4 \%$ & 22 & 10 & 4 & 5 & PROPRAN \\
\hline 54 & Huey LTV & 63,8987 & $63.0 \%$ & $76.6 \%$ & 17 & 11 & 3 & 10 & PROPRAN \\
\hline 55 & Datton SD & 42,6800 & $77.8 \%$ & $64.3 \%$ & 21 & 9 & 5 & 6 & PROPRAN \\
\hline 55 & Zugato STV & 0.0012 & $63.0 \%$ & $71.4 \%$ & 17 & 10 & 4 & 10 & PROPRAN \\
\hline 57 & Zugab LTV & 0.0311 & $74.1 \%$ & $79.6 \%$ & 20 & 11 & 3 & 7 & PROPRAN \\
\hline 58 & OSC & 12.0453 & 81.5\% & $71.4 \%$ & 22 & 10 & 4 & 5 & PROPRAN \\
\hline 59 & SONA & 21.3372 & $77.0 \%$ & $64.7 \%$ & 21 & 9 & 5 & 6 & PROPRAN \\
\hline 60 & FFT TOTAL PON & 5.7684 & $77.6 \%$ & $71.4 \%$ & 21 & 10 & 4 & 5 & PROPRAN \\
\hline 51 & W1 & 5.7644 & $77.6 \%$ & $71.4 \%$ & 21 & 10 & 4 & 6 & PROPRAA: \\
\hline 62 & W2 & 5.7684 & $77.8 *$ & $71.4 \%$ & 21 & 10 & 4 & 6 & PROPRAN \\
\hline 63 & wo & 2.4925 & $77.3 x$ & $69.2 \%$ & 17 & 9 & 4 & 5 & PROPRAN \\
\hline 64 & Noise & 0.0643 & $69.7 x$ & $79.6 \%$ & 16 & 11 & 3 & 11 & PROPRAN \\
\hline 65 & AR HF & 10.7057 & $66.7 \%$ & $64.3 \%$ & 16 & 9 & 5 & 9 & PROPRAN \\
\hline 65 & $A R$ LF & 0.2617 & $74.1 \%$ & $78.6 \%$ & 20 & 11 & 3 & 7 & PROPRAN \\
\hline 67 & AR LF/HF & 0.4004 & $74.1 \%$ & $78.6 \%$ & 20 & 11 & 3 & 7 & PROPRAN \\
\hline
\end{tabular}

Fig. 6. Threshold levels, corresponding sensitivity, specificity, number of true positives TP, true negatives TN, false positives FP and false negative FN cases in different clinical status-discrimination between CHD and control groups (continued)

be interpreted as insufficient power of used ROC method to detect abnormal cases (patients with CHD). The best discrimination between analyzed groups of patients we obtain after propranolol administration in de Haan LTI, Yeh II, Organ BAND, van Geijn ID, OSC and Huey STV indices. First five of them describe pure long term heart rate variability.

\section{LOGISTIC REGRESSION}

Logistic regression finds an equation that best predicts a binary outcome variable from one (or more) predictors [5]. In presented paper we analyzed only prediction based on single 
Fig. 7. Exemplary results of logistic regression for Allan index measured in standing position (code 0 healthy, code 2 - CHD)

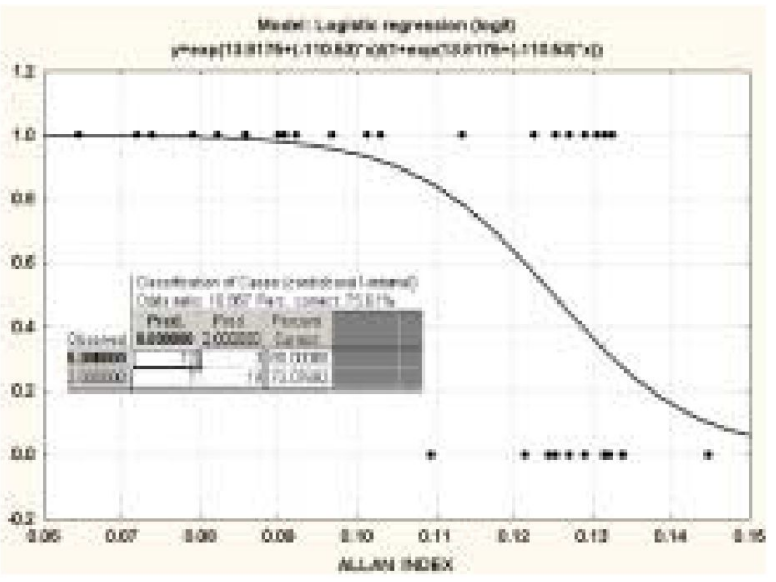

Table 2. Usefulness of analyzed indices in discrimination between CHD and healthy people in five different clinical situations - logistic regression results

\begin{tabular}{lccrrrrl}
\hline \multicolumn{1}{c}{ Parameter } & $p$-value & $\begin{array}{c}\text { Correct } \\
\text { classification } \\
{[\%]}\end{array}$ & TP & TN & FP & FN & STATUS \\
\hline RMSSD & 0.00192 & 69.05 & 21 & 8 & 7 & 6 & SUPINE \\
HUEY LTV & 0.00162 & 75.61 & 24 & 7 & 8 & 2 & STANDING \\
OSC & 0.00107 & 78.05 & 24 & 8 & 7 & 2 & STANDING \\
ALLAN & 0.00002 & 75.61 & 19 & 12 & 3 & 7 & STANDING \\
YEH II & 0.01070 & 73.17 & 26 & 4 & 10 & 1 & PROPRANOLOL \\
ZUGAIB LTV & 0.00476 & 73.17 & 26 & 4 & 10 & 1 & PROPRANOLOL \\
W1 & 0.00223 & 75.61 & 25 & 6 & 8 & 2 & PROPRANOLOL \\
W2 & 0.02720 & 68.29 & 24 & 4 & 10 & 3 & PROPRANOLOL \\
\hline
\end{tabular}

variable. In our case binary dependent variable denotes affiliation either to control group or to CHD group. As a loss function maximum likelihood was used. In all cases Quasi-Newton estimation method with convergence criterion 0.00001 was applied (Fig. 7). We obtained fair results only in eight cases. All other logistic models were statistically not significant. However the general classification quality was between 68 and 78\%, we observed high number of false negatives FN similarly as in ROC results (Table 2). The superiority of logistic method on previous cited is that it allows for parametrization and construction of simple prediction model.

5.

CONCLUSIONS

Three different statistical techniques were used to check usability of single heart rate variability HRV index as a marker in screening test differentiating healthy people with coronary heart disease group. All three methods confirmed that reaction for propranolol administration is prevalent in comparison to other four analyzed clinical situations. It mani- 
fested in changes of rather long-term than short-term heart rate variability. Most promising parameters, which usefulness in discrimination was confirmed simultaneously with all mathematical techniques are: RMSSD in supine position, Huey LTV, OSC and Allan indices in standing position, Yeh II, Zugaib LTV, w1 and w2 after administration of propranolol. However prediction of patients status on the basis of univariate analysis is rather poor. Predominant number of false negative misclassifications suggests that probably use of multivariate approach incorporating combinations of HRV indices and some additional parameters will improve quality of classification. Intentionally no one additional (not HRV) descriptor was used in presented analysis. We want to emphasize, that high degree of biological variability effectively makes impossible diagnosis support based exclusively on single descriptor. Because of small sample size obtained preliminary results should be rather treated as methodology discussion than real medical "gold standards".

\section{References}

[1] J. Moczko, Advanced methods of heart rate signals processing and their usefulness in diagnosis support I. Mathematical heart rate descriptors and virtual instrumentation, Comp. Meth. in Sci. and Tech., 8(2), 65-76, (2002).

[2] M. Hollander, D. A. Wofe, Nonparametric Statistical Methods. John Wiley \& Sons, Inc. (1973).

[3] S. W. Smith, Digital Signal Processing. A practical guide for engineers and scientists. NewnesElsevier Science (2003).

[4] Y. Lu and J.-Q. Fang, Advanced Medical Statistics, World Scientific (2003)

[5] D. W. Hosmer and S. Lemeshow, Applied Logistic Regression, John Wiley \& Sons, Inc. (1989) 